\title{
Effect of Non-uniform Heat Source and Radiation on Transient MHD Flow Past a Vertical Moving Plate with Inclined Magnetic Field and Periodic Heat Flux
}

\author{
Mustafa Rashied Abdullah \\ Faculty of Engineering \\ Al-Ahliyya Amman University \\ Amman, Jordan \\ mrashied@ammanu.edu.jo
}

\author{
Omar K. Alghazawi \\ Faculty of Engineering \\ Al-Ahliyya Amman University \\ Amman, Jordan \\ o.ghazawi@ammanu.edu.jo
}

\author{
Muhammad Al-Ayyad \\ Faculty of Engineering \\ Al-Ahliyya Amman University \\ Amman, Jordan \\ mayyad@ammanu.edu.jo
}

\begin{abstract}
An analysis is carried out in order to examine the effects of non-uniform heat source and thermal radiation on the transient free convective MHD flow past a vertical plate. In this model, an inclined magnetic field with periodic heat flux along the wall is included. The governing equations (in non-dimensional shape) are solved numerically by a fully implicit finite difference scheme. A parametric study is performed in order to illustrate the influences of the model dimensionless parameters, namely, the magnetic parameter, the heat source term, the Grashof number, the Prandtl number, and the radiation parameter. The velocity field and the temperature field are evaluated for several amounts of these parameters. The obtained numerical results are compared with the analytical solution in case of constant heat flux with no heat source, and a full agreement was found.
\end{abstract}

Keywords-free convection; heat source; inclined magnetic field; radiation; periodic heat flux

\section{INTRODUCTION}

There are a lot of recent studies on the effect of magnetohydrodynamics (MHD) on systems of fluid dynamics due to their wide applications in industrial manufacturing processes and cooling systems. Many of these studies were made to predict the velocity and thermal behavior of the boundary layer over a moving vertical plate under different conditions. The effect of heat generation on MHD flow over flat plates has been analyzed in many papers. The effect of temperature dependent heat source on natural convection flow past an accelerated infinite vertical plate of a viscous incompressible fluid under the action of a uniform magnetic field through a porous medium was studied analytically in [1], where the plate temperature was raised linearly with time. Authors in [2] studied the effect of temperature dependent electrical conductivity on steady free convection flow of a viscous incompressible low Prandtl $(\operatorname{Pr} \ll 1)$ fluid past an isothermal vertical plate in the presence of magnetic field and exponentially decaying heat generation. The heat and mass transfer effects on MHD flow of viscous incompressible fluid through a nonhomogeneous porous medium in the presence of heat source, oscillatory suction velocity have been investigated in [3]. Authors in [4] studied the free convection flow in the presence of heat generation and a convective boundary condition. Authors in [5] examined the MHD fluid flow over a vertical porous plate in the presence of radiation and heat generation. Authors in [6] studied the influence of variable viscosity and thermal conductivity on laminar MHD free convective boundary layer flow of a dusty fluid past a vertical porous plate with viscous dissipation, Joule heating, and heat generation. Authors in [7] studied the effect of heat source and chemical reaction on MHD flow along a moving vertical plate with variable temperature. Authors in [8] studied a transient MHD free convective flow over a vertical plate with an impulsive motion in presence of heat generation in a rotating system. An exact solution of a nonlinear MHD flow with heat generation has been studied in [9] for an incompressible viscous fluid along a vertical plate. Authors in [10] studied the transient MHD free convection in a boundary layer flow through a porous medium past a moving infinite vertical plate in presence of heat source and chemical reaction.

The effects of heat flux have been studied extensively. Authors in [11] presented a theoretical study of unsteady free convection flow along a vertical flat plate embedded in a porous medium. The unsteady behavior is computed after the generation of an impulsive heat flux step. The free convection flow of a viscous fluid over a vertical accelerated plate embedded in a porous medium with constant heat flux has been analyzed in [12]. Authors in [13] studied the transient free convection flow of a fluid past an accelerated vertical plate with constant heat flux in the presence of heat generation. An analytical study of transient MHD free convective flow of a viscous incompressible fluid over an infinite vertical plate immersed in a porous medium with viscous dissipation effect and uniform heat flux in the presence of chemical reaction was carried out in [14]. Authors in [15] studied the free convective MHD flow past a moving plate maintained at constant heat flux and embedded in a viscous fluid saturated porous medium. The employed model included the effects of thermal radiation, heat sink and chemical reaction

The effect of inclined magnetic field on the flow and temperature profiles of MHD flow past a vertical plate has also 
been under study. Authors in [16] studied the effects of inclined magnetic field and radiation on the free convective flow of dissipative fluid over a vertical plate through a porous medium in the presence of heat source. The effect of radiation on transient MHD free convection flow of a viscous incompressible fluid past an impulsively moving vertical plate by applying inclined magnetic field was studied in [17]. Authors in [18] examined the effect of inclined magnetic field on the transient flow over a moving vertical plate with variable temperature. The unsteady MHD flow between two nonconducting MHD infinite vertical plates in the presence of uniform inclined magnetic field was considered in [19] where it was assumed that one of the plates was in motion with constant velocity and the other plate was considered to be adiabatic. Authors in [20] studied a theoretical model of transient MHD free convection flow with heat transfer of a viscous, incompressible, radiating and chemically reactive fluid past an impulsively moving vertical plate with ramped heat flux in the presence of inclined magnetic field.

In this paper, the effect of heat generation and thermal radiation on the MHD free convection flow and heat transfer past a vertical plate has been studied. Here, the magnetic field is assumed to be inclined. The contribution of periodic heat flux in the energy equation has been included. The governing non-dimensional differential equations were solved numerically by a fully implicit finite difference scheme known as the Crank-Nicolson method. Numerical computations have been carried out for temperature and velocity profiles. The effects of magnetic parameter, heat source term, Grashof numer, Prandtl number and radiation parameter on the flow behavior and heat transfer process are presented through graphs, and their features are discussed.

\section{MATHEMATICAL FORMULATION}

The problem of laminar, two-dimensional MHD flow of an incompressible electrically conducting viscous fluid past a vertical plate is shown in Figure 1.

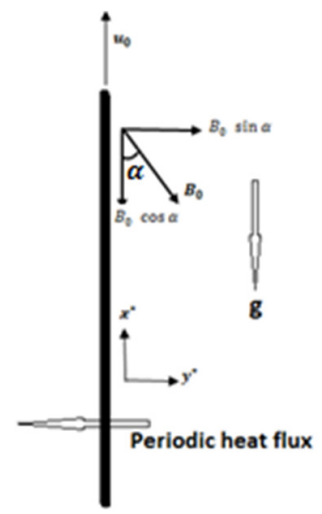

Fig. 1. Schematic diagram of the problem

A conductive liquid with a density $\rho$, dynamic viscosity $\mu$, and electrical conductivity $\sigma$ is considered. Assume the coordinate system to be such that the $x^{*}$-axis is taken along the vertical plate and the $y^{*}$-axis is perpendicular to the plate. The flow is assumed to be laminar and the fluid properties are assumed to be constant. The fluid is subjected to a uniform magnetic field which is assumed to be inclined with an angle relative to the $x^{*}$-axis, and the induced electric and magnetic fields are ignored. At time $t \leq 0$, the plate and the fluid are assumed to be stationary and having the same temperature $T_{\infty}$. At time $t>0$, the plate starts moving with a constant velocity in the presence of a periodic heat flux. Assuming a fully developed flow, the transient governing equations of the MHD flow that represent the fluid motion and temperature are:

$$
\begin{aligned}
& \frac{\partial u^{*}}{\partial t^{*}}=v \frac{\partial^{2} u^{*}}{\partial y^{* 2}}+g \beta\left(T^{*}-T_{\infty}\right)-\frac{\sigma B_{0}^{2}(\sin \alpha)^{2}}{\rho} u^{*} \\
& \frac{\partial T^{*}}{\partial t^{*}}=\frac{k}{\rho c_{p}} \frac{\partial^{2} T^{*}}{\partial y^{* 2}}+\frac{Q_{0}}{\rho c_{p}}\left(T^{*}-T_{\infty}\right)-\frac{\partial q_{r}}{\partial y^{*}}
\end{aligned}
$$

Using the Rosseland approximation for radiation as that given in [21], the radiative heat flux is:

$$
q_{r}=\frac{-4 \sigma^{*}}{3 k^{*}} \frac{\partial T^{* 4}}{\partial y^{*}}
$$

Using Taylor series expansion for $T^{* 4}$, we have:

$$
\frac{\partial T^{* 4}}{\partial y^{*}} \cong 4 T_{\infty}^{3} \frac{\partial T^{*}}{\partial y^{*}}
$$

And then:

$$
\frac{\partial q_{r}}{\partial y^{*}}=\frac{16 \sigma^{*}}{3 k^{*}} T_{\infty}^{3} \frac{\partial^{2} T^{*}}{\partial y^{* 2}}
$$

Using (5), equation (2) becomes:

$$
\frac{\partial T^{*}}{\partial t^{*}}=\frac{k}{\rho c_{p}}\left(1+\frac{16 \sigma^{*}}{3 k^{*} k} T_{\infty}^{3}\right) \frac{\partial^{2} T^{*}}{\partial y^{* 2}}+\frac{Q_{0}}{\rho c_{p}}\left(T^{*}-T_{\infty}\right)
$$

where $u^{*}$ is the axial velocity, $T^{*}$ is the fluid temperature, $v$ is the kinemetic viscosity, $k$ is the thermal conductivity, and $C_{p}$ is the speicific heat. With initial and boundary conditions:

$$
\begin{aligned}
t^{*} \leq 0: u^{*} & =0, T^{*}=T_{\infty} \text { for all } \mathrm{y}^{*} \\
t^{*}>0: u^{*} & =u_{0}, \frac{\partial T^{*}}{\partial y^{*}}=-\frac{q}{k} \cos \left(\omega^{*} t^{*}\right) \text { at } \mathrm{y}^{*}=0, \\
u^{*} & \rightarrow 0, T^{*} \rightarrow T_{\infty}, \text { as } y^{*}=\infty
\end{aligned}
$$

To write the governing equations in dimensionless form, the following dimensionaless variables are introduced:

$$
\begin{aligned}
& y=\frac{y^{*} u_{0}}{v}, \quad t=\frac{t^{*} u_{0}^{2}}{v}, \omega=\frac{\omega^{*} v}{u_{0}^{2}}, u=\frac{u^{*}}{u_{0}} \\
& \theta=\frac{k\left(T-T_{\infty}\right) u_{0}}{q v}, \quad M=\frac{\sigma B_{0}^{2} v}{\rho u_{0}^{2}}, G r=\frac{g \beta q v^{2}}{k u_{0}}, \operatorname{Pr}=\frac{\mu c_{p}}{k} \\
& R=\frac{16 \sigma^{*} T_{\infty}^{3}}{3 k^{*} k} \quad S=\frac{Q_{0} q v}{\rho c_{p} k u_{0}}
\end{aligned}
$$

The dimensionless equations become:

$$
\begin{aligned}
& \frac{\partial u}{\partial t}=\frac{\partial^{2} u}{\partial y^{2}}+\operatorname{Gr} \theta-M(\sin \alpha)^{2} u \\
& \frac{\partial \theta}{\partial t}=\frac{(1+R)}{P r} \frac{\partial^{2} \theta}{\partial y^{2}}+S \theta
\end{aligned}
$$

The dimensionless boundary conditions can be written as: 


$$
\begin{aligned}
t \leq 0: u & =0, \theta=0 \text { for all } \mathrm{y}^{*} \\
t>0: u & =1, \frac{\partial \theta}{\partial y}=-\cos (\omega t) \text { at } \mathrm{y}=0, \\
u & \rightarrow 0, \theta \rightarrow 0, \text { as } y=\infty
\end{aligned}
$$

\section{NUMERICAL ANALYSIS}

The dimensionless momentum and energy equations subject to the initial and boundary conditions are solved numerically by using a stable fully implicit finite difference technique. Using the Crank-Nicolson method, the fluid region is divided into a grid in $x$ and $y$ directions and central finite divided difference equations are obtained at each time step. To determine the velocity and temperature distributions for different parameter values the Thomas algorithm is used by solving the resulting finite difference equations with tridiagonal coeffecient matrix.

\section{EXACT SOLUTION}

The numerical solution has been verified by comparison with the analytical solution in the case of constant heat flux without heat generation. The eigenfunction expansion method was used to solve the special form of the energy equation. The dimensionless time dependent energy equation can be written as:

$$
\frac{\partial \theta}{\partial t}=\frac{(1+R)}{P r} \frac{\partial^{2} \theta}{\partial y^{2}}
$$

The boundary conditions for constant heat flux can be written as follows:

$$
\begin{aligned}
t \leq 0: & \theta=0 \text { for all } y \\
t>0: & \frac{\partial \theta}{\partial Y}=-1 \text { at } y=0, \\
& \theta \rightarrow 0, \text { as } y=\infty
\end{aligned}
$$

Since the above boundary conditions are non homogeneous, a new function is introduced to convert them to homogeneous :

$$
H(y, t)=\theta(y, t)-(\infty-y)
$$

Applying (14), the energy equation becomes:

$$
\frac{\partial H}{\partial t}=\frac{(1+R)}{P r} \frac{\partial^{2} H}{\partial y^{2}}
$$

And the boundary conditions can be written as:

$$
\begin{gathered}
t \leq 0: H=(y-\infty) \text { for all } \mathrm{y} \\
t>0: \frac{\partial H}{\partial y}=0 \text { at } \mathrm{y}=0, \\
H \rightarrow 0, \text { as } y=\infty
\end{gathered}
$$

Let $H(y, t)=\varnothing(y) \delta(t)$ and compute the derivatives and substitute into (15) to obtain the eigenvalue problem as:

$$
\frac{d^{2} \emptyset}{d y^{2}}+\lambda \emptyset=0 \quad \frac{\partial \phi}{\partial y}(0)=\emptyset(L)=0
$$

where $\lambda$ is the separation constant, and $L \rightarrow \infty$. The above equation has the following solution:

$$
\emptyset_{n}(y)=\cos (\sqrt{\lambda} y)
$$

with eigenvalues:

$$
\lambda_{n}=\left(\frac{(2 n-1) \pi}{2 L}\right)^{2}
$$

For each $\mathrm{n}$, the solution for $\delta(t)$ is $\delta_{n}(t)=e^{-\frac{\lambda_{n}(1+R) t}{p r}}$, hence the series solution for $H(y, t)$ becomes:

$$
H(y, t)=\sum_{n=1}^{\infty} A_{n} \cos (\sqrt{\lambda} y) e^{-\frac{\lambda_{n}(1+R) t}{p r}}
$$

which satisfies the non-homogeneous initial condition: $H(y, 0)=(y-\infty)$. Hence:

$$
A_{n}=\frac{\frac{1}{\lambda}(\cos (\sqrt{\lambda} L)-1)}{\left(\frac{L}{2}+\frac{\sin (2 \sqrt{\lambda} \mathrm{L})}{4 \sqrt{\lambda}}\right)} \quad \text { where } n=1,2, \ldots \ldots, \infty \text {. The final }
$$

form of solution becomes:

$$
\begin{gathered}
\theta(y, t)=\left(\sum_{n=1}^{\infty} \frac{\frac{1}{\lambda}(\cos (\sqrt{\lambda} L)-1)}{\left(\frac{L}{2}+\frac{\sin (2 \sqrt{\lambda} L)}{4 \sqrt{\lambda}}\right)} \cos (\sqrt{\lambda} y) e^{-\frac{\lambda_{n}(1+R) t}{p r}}\right)+(\infty-y)(21) \\
\text { V. RESULTS AND DISCUSSION }
\end{gathered}
$$

Using the above numerical method, the governing equations of the problem are solved for various sets of values of the physical parameters. The numerical results obtained are presented for dimensionless velocity and dimensionless temperature in Figures 1-12. The velocity profile $u$ is presented for different values of dimensionless parameters. The following dimensionless variables are used to get the results: $\mathrm{Gr}=5, \mathrm{Pr}=7$, $M=1, \omega=\pi / 4, R=2, S=0.5, \alpha=\pi / 3$ and $t=1$. The dimensionless velocity $u$ increases from 1 at the plate, reaching its maximum value near the plate and decreases to 0 at the free stream. Figure 2 shows the dimensionless velocity $u$ for various values of Grashoff number Gr. The velocity profiles show that the velocity increases with an increase in Gr. It is also shown that the velocity increases nearby the plate for higher $G r$ more than for lower Gr.

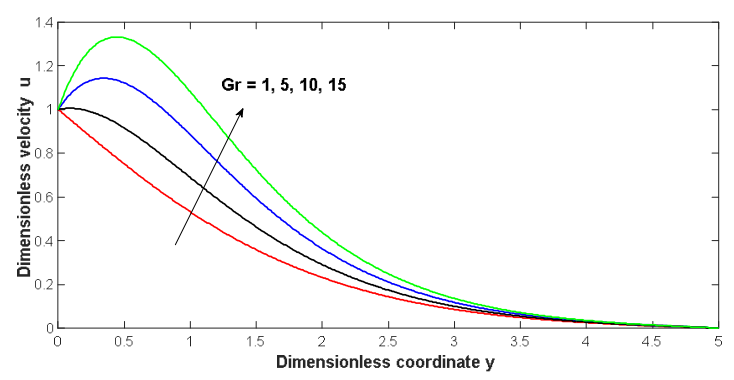

Fig. 2. Velocity profile for different Grashof numbers (Gr)

Figure 3 demonstrates the effect of magnetic number $M$ on the velocity. It is observed that the increase in $M$ decreases the velocity profile. This means that increasing the magnetic field tends to slow the fluid flow. It is also shown that retardation of the flow can be achieved by law values of magnetic number. The effect of Prandtl number on velocity and temperature behavior is illustrated in Figures 4 and 5. Increasing Pr means decreasing thermal conductivity, and therefore reducing heat transfer rate. Hence the effect of Pr is to decrease velocity and 
temperature. The profiles were obtained for different liquids. It is clear that for high $\operatorname{Pr}$ the velocity and temperature magnitudes will be reduced significantly. Figure 6 shows the effect of angle of inclination of the magnetic field $\alpha$. It is noticed that increasing $\alpha$ will increase the perpendicular component of magnetic field and hence decrease the velocity.

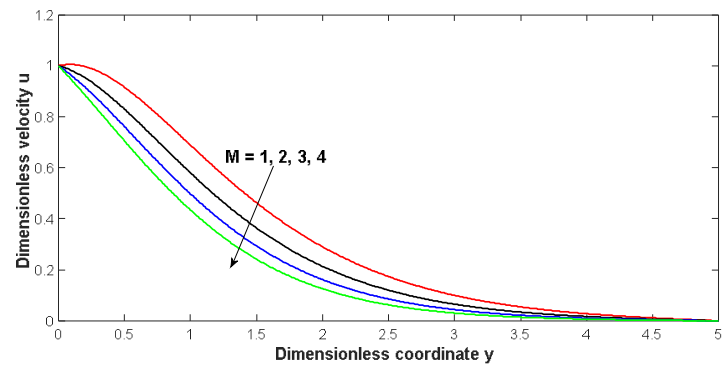

Fig. 3. Velocity profile for different values of magnetic parameter $(M)$

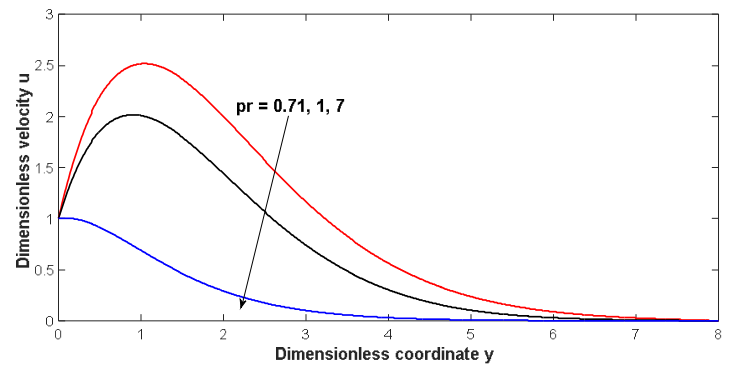

Fig. 4. Velocity profile for different Prandtl numbers (Pr)

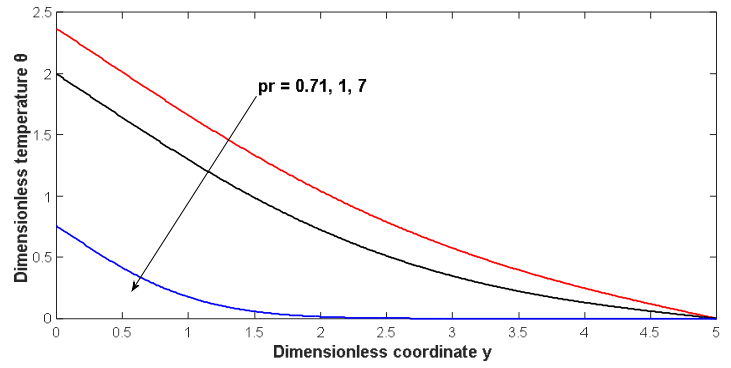

Fig. 5. Temperature profile for different Prandtl numbers (Pr)

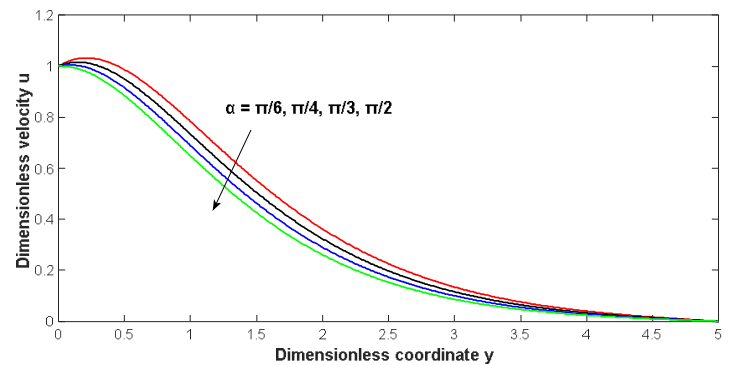

Fig. 6. Velocity profile for different magnetic field inclination angles $(\alpha)$

The effect of the heat source term $S$ on the velocity and temperature profiles is illustrated in Figures 7 and 8 . The velocity will increase as the heat source increases especially in the area around the plate. It is noticed that increasing the heat source will increase the plate and fluid temperatures. The temperature profile for different values of radiation parameter $R$ is expressed graphically in Figure 9. Increasing $R$ enhances the fluid temperature. Figures 10 and 11 depict the velocity and temperature profiles for different time values. It is proven that increasing time will increase the fluid velocity and temperature of the plate and fluid.

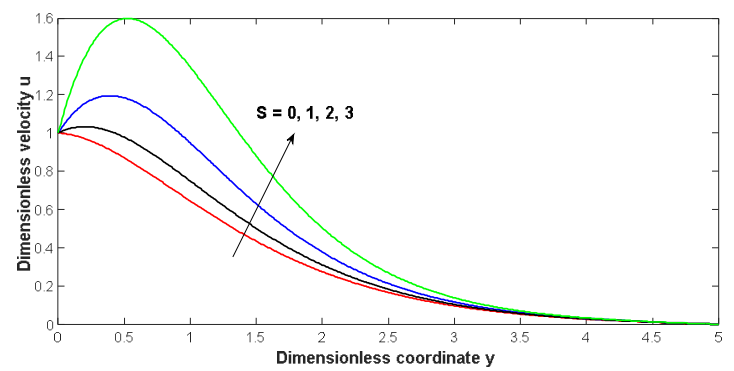

Fig. 7. Velocity profile for different values of heat source term $(S)$

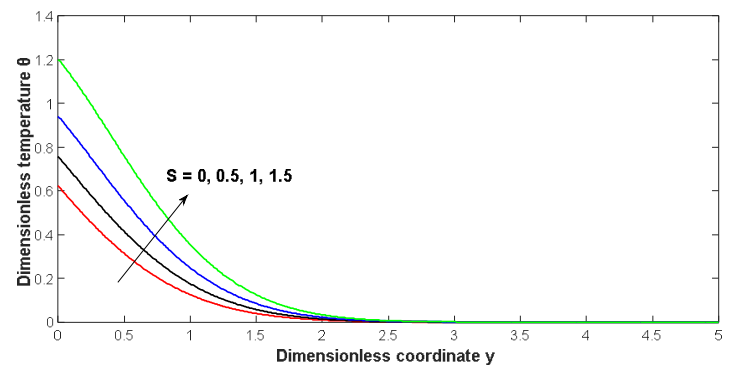

Fig. 8. Temperature profile for different values of heat source term $(S)$

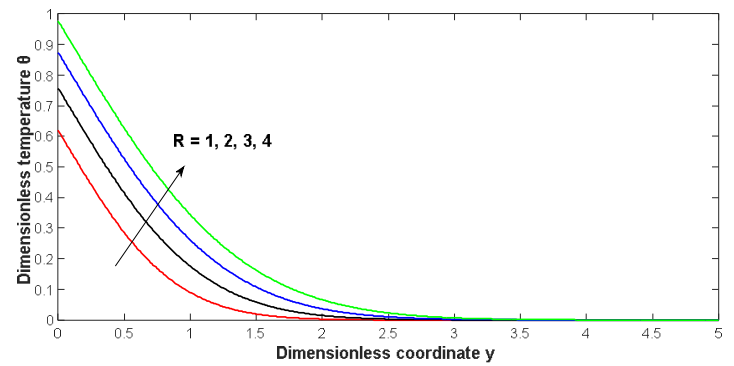

Fig. 9. Temperature profile for different radiation parameters $(R)$

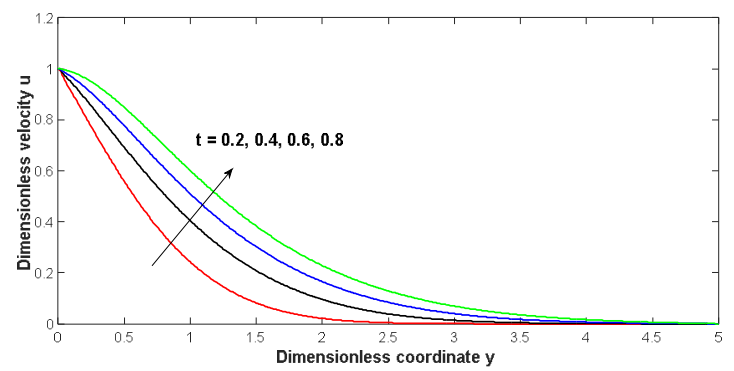

Fig. 10. Velocity profile for different time values $(t)$ 


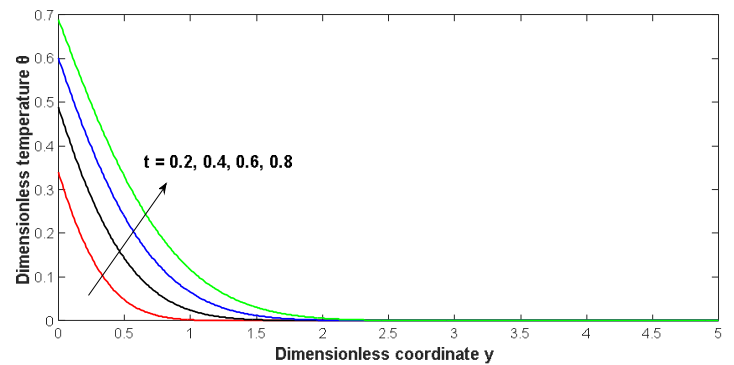

Fig. 11. Temperature profile for different time values $(t)$

Figure 12 illustrates the effect of the heat flux frequency on the transient plate temperature behavior where the time is taken up to $t=5$. It is observed that the fluctuating behavior of the heat flux is reflected on the temperature behavior. A comparison between the numerical implicit solution and the exact solution for the dimensionless temperature in case of constant heat flux and in the absence of heat generation is shown in Figure 13. It is observed that the two solutions are in full agreement.

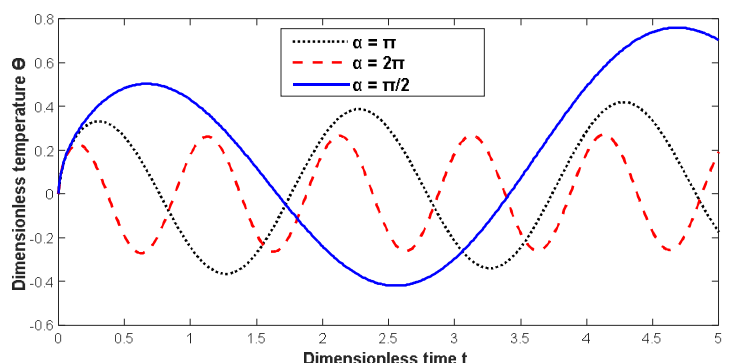

Fig. 12. Transient temperature for different values of heat flux frequency

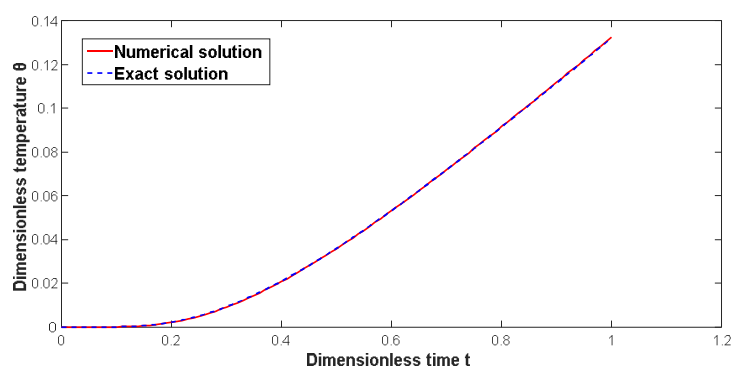

Fig. 13. Comparison of temperature numerical and analytical results

\section{CONCLUSIONS}

In this paper, a fully implicit numerical solution to the problem of transient free convective laminar flow past a moving vertical plate in the presence of periodic heat flux and inclined magnetic field has been presented. The velocity and temperature behaviors were studied for different dimensionless parameters by applying the Crank-Niclson technique, which is verified by an eigenfunction expansion method for constant heat flux, were computed. The effect of different parameters such as magnetic parameter with inclination angle, Grashof number, Prandtl number, heat source parameter, radiation parameter and time were studied. Some of the conclusions of this work are:
- The effect of MHD on the fluid flow appears through the magnetic parameter effect. Magnetic field exerts a retarding influence on the fluid velocity.

- Increasing the Grashof number will cause an increase of the velocity.

- Increased heat source increases velocity and temperature.

- Increasing the Prandtl number will decrease velocity and temperature.

- Thermal radiation tends to enhance the plate and fluid flow temperature.

- Inclination angle of magnetic field will influence the fluid flow.

- The fluctuating behavior of the heat flux is reflected on the temperature behavior.

\section{NOMENCLATURE}

Bo Magnetic flux density

$C p \quad$ Specific heat

Gr Grashof number

$k$ thermal conductivity

$k^{*} \quad$ Mean absorption coefficient

$M \quad$ Magnetic parameter

Pr Prandtl number

$q \quad$ Constant heat flux at the surface

$q_{r} \quad$ Radiative heat flux

$Q_{0} \quad$ Heat generation coefficient

$R \quad$ Radiation parameter

$S \quad$ Heat generation parameter

$t^{*} \quad$ Time

$t \quad$ Dimensionless time

$T^{*} \quad$ Temperature

$T_{\infty} \quad$ Free stream temperature

$u^{*} \quad$ Velocity component in the $\mathrm{x}$ direction

$u$ Dimensionless velocity

$x^{*}, y^{*}$ Cartesian coordinates

$y$ Dimensionless coordinate

$\omega^{*} \quad$ Frequency of oscillation

$\omega$ Dimensionless frequency

$\alpha \quad$ Inclination angle of the magnetic field

$\beta \quad$ Volumetric coefficient of thermal expansion

$\varphi, \delta \quad$ Separation variables

$\theta \quad$ Dimensionless temperature

$\lambda$ Separation constant

$v \quad$ Kinematic viscosity

$\sigma \quad$ Electrical conductivity

$\sigma^{*} \quad$ Stefan-Boltzmann constant

$\rho$ Density

$\mu \quad$ Dynamic viscosity

\section{REFERENCES}

[1] V. Rajesh, S. V. K. Varma, "Heat source effects on MHD flow past an exponentially accelerated vertical plate with variable temperature through a porous medium", International Journal of Applied Mathematics and Mechanics, Vol. 6, No. 12, pp. 68-78, 2010 
[2] P. R. Shrama, G. Singh, "Steady MHD natural convection flow with variable electrical conductivity and heat generation along an isothermal vertical plate", Tamkang Journal of Science and Engineering, Vol. 13, No. 3, pp. 235-242, 2010

[3] V. Ravikumar, M. C. Raju, G. S. S. Raju, "Heat and mass transfer effects on MHD flow of viscous fluid through non-homogeneous porous medium in presence of temperature dependent heat source", International Journal of Contemponary Mathematical Sciences, Vol. 7, No. 32, pp. 1597-1604, 2012

[4] R. S. Yadav, P. R. Sharma, "Analysis of MHD convective flow along a moving semi vertical plate with internal heat generation", International Journal of Engineering Research \& Technology, Vol. 3, No. 4, pp. 23422348, 2014

[5] P. Mangathai, G. V. R. Reddy, B. R. Reddy, "MHD free convective flow past a vertical porous plate in the presence of radiation and heat generation”, International Journal of Chemical Sciences, Vol. 14, No. 3, pp. 1577-1597, 2016

[6] G. Hazarika, J. Konch, "Effects of variable viscosity and thermal conductivity on magnetohydrodynamic free convection dusty fluid along a vertical porous plate with heat generation", Turkish Journal of Physics, Vol. 40, No. 1, pp. 52-68, 2016

[7] P. K. Rout, S. N. Sahoo, G. C. Dash, "Effect of heat source and chemical reaction on MHD flow past a vertical plate with variable temperature", Journal of Naval Architecture and Marine Engineering, Vol. 13, No. 1, pp. 1813-8235, 2016

[8] U. S. Rajput, M. Shareef, "Unsteady MHD flow past impulsively started vertical plate in porous medium with heat source and chemical reaction", International Journal of Chemical Sciences, Vol. 15, No. 3, pp.1-13, 2017

[9] J. D. Olisa, "Transient laminar MHD free convective heat transfer past a vertical plate with heat generation", The International Journal of Engineering and Science, Vol. 6, No. 4, pp. 8-13, 2017

[10] M. V. Krishna, M. G. Reddy, "MHD Free Convective Boundary Layer Flow Through Porous Medium Past a Moving Vertical Plate with Heat Source and Chemical Reaction", International Conference on Processing of Materials, Minerals and Energy, Andhra Pradesh, India, July 29-30, 2016

[11] J. J. Shu, I. Pop, “Transient conjugate free convection from a vertical flat plate in a porous medium subjected to a sudden change in surface heat flux", International Journal of Engineering Science, Vol. 36, No. 2, pp. 207-214, 1998

[12] R. C. Chaudhary, M. C. Goyal, A. Jain, "Free convection effects on MHD flow past an infinite vertical accelerated plate embedded in porous media with constant heat flux", Matematicas: Ensenanza Universitaria, Vol. 17, No. 2, pp.73-82, 2009

[13] M. Narahari, L. Debnath, "Unsteady magnetohydrodynamic free convection flow past an accelerated vertical plate with constant heat flux and heat generation or absorption", Journal of Applied Mathematics and Mechanics, Vol. 93, No. 1, pp. 38-49, 2013

[14] R. M. Ramana, J. G. Kumar, "Influence of chemical reaction on unsteady MHD free convective flow past a vertical plate with uniform heat and mass flux through porous medium", International Journal of Advanced Research in Computer Engineering \& Technology, Vol. 5, No. 5, pp. 1400-1407, 2016

[15] M. A. E. Aziz, A. S. Yahya, "Heat and mass transfer of unsteady hydromagnetic free convection flow through porous medium past a vertical plate with uniform surface heat flux", Journal of Theoretical and Applied Mechanics, Vol. 47, No. 3, pp. 25-58, 2017

[16] N. Sandeep, V. Sugunamma, "Effect of inclined magnetic field on unsteady free convective flow of dissipative fluid past a vertical plate", World Applied Sciences Journal, Vol. 22, No. 7, pp. 975-984, 2013

[17] N. Sandeep, V. Sugunamma, "Radiation inclined magnetic field effects on unsteady hydromagnetic free convection flow past an impulsively moving vertical plate in a porous medium", Journal of Applied Fluid Mechanics, Vol. 7, No. 2, pp. 275-286, 2014

[18] N. K. Singh, V. Kumar, G. K. Sharma, "The effect of inclined magnetic field on unsteady flow past on moving vertical plate with variable temperature", International Journal of Latest Technology in Engineering, Management \& Applied Science, Vol. 5, No. 2, pp. 34-37, 2016

[19] P. T. Hemamalini, M. Shanthi, "Heat and mass transfer on unsteady MHD flow in tow non-conducting infinite vertical parallel plates with inclined magnetic field", International Journal of Mechanical Engineering and Technology, Vol. 9, No. 3, pp. 521-536, 2018

[20] G. S. Setha, P. K. Mandal, A. J. Chamkha, "MHD free convective flow past an impulsively moving vertical plate with ramped heat flux through porous medium in the presence of inclined magnetic field", Frontiers in Heat and Mass Transfer, Vol. 7, No. 23, pp. 1-12, 2016

[21] E. M. Sparrow, R. D. Cess, Radiation Heat Transfer, Augmented Edition, Hemisphere Publishing Corporation, 1978 